\title{
PREPARO DA FAMÍLIA PARA A REVELAÇÃO DO HIV NA INFÂNCIA: EXPERIÊNCIAS DE ENFERMEIRAS
}

\author{
Renata de Moura Bubadué 1 e Ivone Evangelista Cabral 2
}

${ }_{1}$ Faculdade de Ciências e Educação Sena Aires (FACESA), Brasil. renatabubadue@gmail.com 2Conselho Nacional de Desenvolvimento Científico e Tecnológico (CNPq) Ní/ EEAN/UFRJ, Brasil. Icabral444@gmail.com

\begin{abstract}
Resumo. A participação da enfermeira/o no processo de revelação do HIV/AIDS na infância é pouco explorado na literatura. Objetivos: desvelar a experiência de enfermeiras no preparo de familiares para revelar condição HIV soropositiva da criança. Métodos: Pesquisa qualitativa desenvolvida com o Método Criativo e Sensível, com 13 enfermeiras no Rio de Janeiro, Brasil. Dados tratados com análise de discurso de Orlandi. Resultados: Os discursos de preconceito e estigma estrutural representam obstáculos à participação da enfermeira no processo de preparo de familiares para revelar o HIV/aids à criança que é soropositiva. Conclusões: Reconhece-se o direito de a criança ter acesso a informação sobre sua condição, desde que de forma segura e numa linguagem clara e atraumática, o que requer um preparo prévio de quem fará a revelação.
\end{abstract}

Palavras-chave: HIV; Revelação da Verdade; Enfermagem Pediátrica.

\section{PRAPARATION OF THE FAMILY TO DISCLOSE HIV DURING CHILDHOOD: NURSES' EXPERIENCES}

\begin{abstract}
Nurse's participation in the process of HIVIAIDS disclosure during childhood is not very explored in the literature. Objectives: to unveil the experience of nursing on the preparation of families to disclose HIV to the sereopositive child. Methods: Qualitative research developed through the Creativity and Sensitivity Method with 13 nurses in Rio de Janeiro, Brazil. Data was submitted to Orlandi's discourse analysis. Results: Discourses of structural prejudice and stigma represent obstacles to the nurse's participation in the process of preparing families to reveal HIV/ AIDS to children who are HIV positive. Conclusions: Children's right to access information about their condition is recognized, as long as it is in a safe way with clear and non-traumatic language. This requires previous preparation from those who will disclose it.
\end{abstract}

Keywords: HIV; Truth Disclosure; Pediatric Nursing.

\section{INTRODUÇÃO}

Historicamente, o HIV/AIDS é uma condição de saúde comum entre adultos com vida sexualmente ativa e sem proteção. Contudo, há um número significativo de crianças vivendo com HIV/AIDS, pois de acordo com o Fundo das Nações Unidas para a Infância (UNICEF) há três milhões de crianças (0-18 anos) vivendo com HIV. Destas, 39 mil moram no Brasil, o país da América Latina com o maior índice de infecção nessa população, sendo que a maior parte foi infectada pela via vertical, quando a criança é infectada durante a gestação ou parto. (Brasil, 2018; Unicef, 2019)

Esse grupo de crianças apresenta necessidades de saúde especiais e singulares, cujas demandas de cuidados cotidianos ou habituais modificados incluem a revelação do HIV. O 
manejo da terapia antirretroviral, como parte do cuidado medicamentoso, e a comunicação de notícias difíceis têm despertado maior interesse dos estudos (Potrich, Paula, Padoin, \& Gomes, 2016; Adedimeji et al., 2017), quando comparado a estudos sobre preparo da família na revelação à criança de sua condição HIV soropositiva. No Brasil, há um consenso de que as crianças têm direito de participar no seu cuidado de saúde. Todavia, no contexto do HIV por infecção vertical, essa participação acontece de forma mais ativa após tomarem conhecimento de sua condição de saúde. Os responsáveis por fornecer essa informação à criança são os seus responsáveis legais (Bubadué et al., 2016). Para isso, é preciso que eles sejam preparados por uma equipe multiprofissional, a qual inclui a Enfermeira.

As Enfermeiras têm responsabilidade ética e legal com o cuidado de crianças e suas famílias em todas as suas necessidades de maneira integral e promovendo a inclusão e escuta atenta de todos envolvidos no processo (Bubadué \& Cabral, 2019). Entretanto, pesquisas de campo e documentos ministeriais disponíveis sobre o preparo da família para a revelação têm focado mais no papel de médicos e psicólogos; e não na enfermeira (Brasil, 2017, 2018; WHO, 2011; Gyanfi et. al., 2017; Wright et al., 2017; Sariah et al., 2016; van Elsland et al., 2019).

Diante desse contexto, questiona-se: Quais são as experiências de formação e de prática profissional construídas pelas enfermeiras sobre o preparo da família para revelar o HIV na infância? Tem-se por objetivo desvelar experiências de formação e prática de participação de enfermeiras no preparo de familiares para que revelem a condição HIV soropositiva à criança.

\section{METODOLOGIA}

Estudo exploratório e interpretativo, desenvolvido com dados primários produzidos com o método criativo sensível (MCS) (Cabral \& Neves, 2016) de pesquisa baseada em arte (PBA) (McNiff, 2007; Archibald, Caine, \& Scott, 2017). O MCS fundamenta-se na prática dialógica freiriana de condução de pesquisa grupal, com inversão da dinâmica de poder do pesquisador para o participante de pesquisa, ao proporcionar um espaço seguro para discussões grupais. Inspira-se nos círculos de cultura (Freire, 2006) ao reconhecer a agência do participante de pesquisa na interação grupal, por meio de atividades que estimulam a reflexão e construção do conhecimento. 
$\mathrm{Na}$ PBA, a arte proporciona uma via de acesso a subjetividade e alteridade humana, um canal de comunicação sensível, com liberdade para a pessoa expressar suas percepções, impressões e sentimentos que as palavras verbalmente manifestas poderiam ser limitadas. As palavras ditas nem sempre conseguem incorporar o sentido da experiência vivencial. (McNiff, 2007) Particularmente relevante para a abordagem de temas sensíveis, ou seja, aqueles que apresentam elevada carga de estigma e preconceito, porque sua construção social está associada a sentimentos negativos que provocam desconfortos e constrangimentos. (Partelli \& Cabral, 2019; Caldas, Dias, Sousa, \& Teixeira, 2019).

Nesse método, o pesquisador de campo escolhe uma dinâmica de criatividade e sensibilidade (DCS) que seja capaz de mobilizar experiências e saberes do participante, respondendo a questão geradora de debate, sob a forma de produções do tipo artística (PA). Essas PA são dispositivos metafóricos materializados em desenhos, histórias, pintura, dramatização, fotografia, recorte e colagem etc representativos de situações existenciais concretas. O pesquisador assume o papel de animador grupal ao conduzir a discussão coletiva, codificação e descodificação de temas geradores de debates. (Soratto et al., 2014; Cabral \& Neves, 2016).

\subsection{A Dinâmica de Criatividade e Sensibilidade e seus procedimentos}

Tendo em vista que a arte funciona como um canal minimizador de estresse ao revitalizar experiências latentes sobre o HIV/aids na infância, elegeu-se a dinâmica de criatividade e sensibilidade (DCS) linha da vida profissional. Sua operacionalização aconteceu em seis encontros grupais, contando-se com a participação de duas a três enfermeiras, em dois momentos diferentes (Agosto/2014 e Janeiro/2015), para não interferir na dinâmica de atendimento do serviço, totalizando 13 participantes.

Para o MCS (Cabral \& Neves, 2018), o quantitativo mínimo de participantes é determinado pela representatividade de vozes presentes no discurso de cada uma, as experiências socialmente construídas e as interações com outras pessoas que enriquecem essas experiências. No diálogo, é necessário a expressão do EU-TU que traduzem as vozes de outras pessoas que foram internalizadas para constituir a alteridade. Nesse sentido, minimamente duas pessoas, com sua polifonia de vozes, são essenciais para o desenvolvimento da DCS do MCS e a produção de sentidos do objeto de investigação que 
está sendo conduzido. Cada encontro da DCS teve um tempo médio de, aproximadamente, 54 minutos.

Como recomendado pelo MCS, a DSC foi implementada em cinco momentos. No primeiro, do acolhimento e apresentação, o animador grupal (pesquisador de campo) e um auxiliar de pesquisa receberam os participantes à porta da sala, cumprimentando-os e introduzindo-os no ambiente. Cada pessoa escreveu em um pedaço de papel, o nome que gostaria de ser identificado durante a DCS e o afixou em lugar visível. Se acomodaram em semicírculo em frente a uma linha de barbante estendida no canto da sala e cada pessoa se apresentou. No segundo momento, o animador explicou a DCS aos participantes, seus objetivos, o tempo que dispunham para completar os espaços vazios da linha da vida profissional em resposta às seguintes questões geradoras de debate (QGD): Na sua trajetória profissional, qual sua experiência no preparo de famílias para revelar o HIV à criança? A segunda etapa consistiu na resposta à QGD em um produção artística coletiva. No terceiro e quarto momentos, a contribuição de cada participante à linha da vida profissional, coletivamente elaborada, foi apresentada, ao mesmo tempo em que sua refletiam sobre o que escreveram, destacando as similaridades e as diferenças. Esse procedimento no MCS é denominado de codificação gradual dos significados individuais e coletivos em temas e subtemas, para posterior indexação do pesqusisador ao tema gerador. Esses temas foram apresentados aos participantes na quinta etapa para que houvesse aprofunamento da reflexão crítica, recodificação e elaboração da síntese temática.

Os registros verbais foram gravados em áudio usando-se um aplicativo do aparelho celular móvel, após autorização das participantes. O auxiliar de pesquisa fez as anotações de campo cujos registros foram somados aos áudios transcritos na integra.

\subsection{Participantes}

Os critérios de seleção dos participantes foram ser enfermeiras com experiência assistencial de, no mínimo, um ano e que atenderam criança com HIV e suas famílias em algum momento da sua vida profissional, independente do sexo, que atuaram em serviços de referência para atendimento a criança com HIV positivo, na cidade do Rio de Janeiro, e que se expuseram a processos de educação continuada e permanente ao longo de sua trajetória profissional. Os participantes enfermeiros/as foram captados em serviços de referência para 
tratamento ambulatorial de criança com HIV/aids na rede pública do Sistema Único de Saúde. O total de participantes foi de 13 enfermeiras.

\subsection{Cenário}

O estudo foi desenvolvido no cenário da comunidade na cidade do Rio de Janeiro, sendo flexibilizado o local de produção de dados de acordo com a preferência e disponibilidade das participantes do estudo.

\subsection{Tratamento e Análise de dados}

O material empírico registrado por meio da gravação foi transcrito na íntegra e subemetido à análise de discurso proposta por Orlandi (2013), em que a linguagem é parte de um movimento social constitutivo do ser humano e sua história. O significado da linguagem é ordenado pelo insconsciente e pela ideologia, mesmo que ela não se apresente explicitamente.

$\mathrm{Na}$ primeira etapa, o material empírico bruto foi lido e sistematizado para conferir inteligibilidade ao texto, aplicando-se a estratégia de dessuperficialização do corpus textual. Essa material é submetido à marcação do tempo de enunciação de cada participante, à identificação das primeiras pistas dos dispositivos analíticos; ou seja, recursos linguísticos (paráfrase, metáfora, polifonia, etc.) que anunciam a formação discursiva de quem fala, de onde fala e para qual audiência essa fala se dirige. Ao proceder desse modo a dessuperficialização, o texto é reordenado para constituir um fio conductor de sentido que contribui para a interpretação de como o discurso funciona e qual é a formação discursiva que circula entre e a partir dos enunciados.

Compreende-se formação discursiva como a construção ideológica, que determina o que pode ser dito e o que deve ser dito em uma certa conjuntura histórico-social implícita à produção do discurso. Portanto, não é estática e pode sofrer mudanças ao longo do tempo para convergir com o momento politico e histórico dos produtores do discurso (Orlandi, 2013). Essas formações são constituídas por diversos objetos discursivos que culminam num processo discursivo; formações ideológicas que direcionam os participantes em suas relações com o tema. Os discursos das enfermeiras foram alinhados com os marcadores temporais utilizados na DCS, demarcando-os desde sua formação acadêmica até o momento atual que se encontravam na época em que a produção de dados foi 
implementada. Eles foram linguisticamente sistematizados com acontecimentos na forma de situações existencias concretas. Por fim, os dispositivos analíticos aplicados aos discursos constituíram-se em objetos discursivos, que foram aproximados por sentidos similares e diferentes para constituir a formação discursive, imaginária e ideológica. Com isso, após a codificação dos objetos discursivos, procedeu-se a indexação deles em três temas: formação discursiva e ideológica de enfermeiras nas décadas de 1980-2010; a formação profissional para o cuidado à criança com HIV/aids; e, enfermeiras na revelação do HIV/aids à criança

\subsection{Critérios de encerramento do trabalho de campo}

$\mathrm{Na}$ análise de discurso proposta por Orlandi (2013) há três elementos centrais que contribuem para a interrupção do trabalho de campo: a) o efeito de significados constituído pelos participantes quando usam diferentes dispositivos linguísticos (metáfora, paráfrase, interdiscurso, silêncio constitutivo, etc.) para construir significados semelhantes; b) similaridade nos contextos de enunciação (intergeracional e emersão do HIV na contemporaniedade); e c) coesão dos objetos discursivos para apontar as formações ideológicas das enfermeiras.

\subsection{Considerações éticas}

Pesquisa aprovada pelo Comitê de Ética em Pesquisa da Escola de Enfermagem Anna Nery (EEAN/UFRJ) e Instituto de Atenção São Francisco de Assis/HESFA, sob o protocolo n. 1.515.557 em consonância com a Resolução nำ466/2012, do Conselho Nacional de Saúde, referente à regulamentação de pesquisas envolvendo seres humanos. Obteve-se a assinatura do Termo de Consentimento Livre e Esclarecido, de todas as participantes antes de iniciar o encontro grupal agendado para o dia.

\section{RESULTADOS}

As participantes possuíam idades de 25 a 51 anos, trabalhavam no sistema público de saúde no Rio de Janeiro, uma delas concluiu o curso de graduação em Enfermagem na década de 1980, nove nos anos 2000 e uma na década de 2010. Oito eram especialistas em Enfermagem em Saúde da Criança e do Adolescente, três eram doutoras e três estavam cursando o doutorado; oito eram Mestres em Enfermagem e duas estavam cursando o Mestrado. A linha da vida profissional mostra que a participação das enfermeiras no preparo 
de familiares, para revelar a condição HIV à criança soropositiva, é socialmente construída por um Eu, simultaneamente social e profissional. Suas experiências em cada marco temporal (antes, durante e após a graduação como enfermeira) estabelece nexos com o modo como o HIV/aids era abordado pelos meios de comunicação em cada época.

\subsection{Formação discursiva e ideológica de enfermeiras nas décadas de 1980- 2010}

A formação das enfermeiras ocorreu entre os anos de 1981 e 2011 e suas vidas profissionais começaram nos anos 1986 a 2012. Todavia, a experiência com pessoas infectadas pelo HIV inicia-se na vida social, antes da formação profissional, quando suas formações ideológicas estavam impregnadas de estigma, preconceito e desinformação. $\mathrm{Na}$ linha da vida profissional das enfermeiras, o tema HIV assume diferentes efeitos de sentido em três épocas. A primeira, antes de 1983, quando não havia o debate público sobre o tema no Brasil; a segunda, entre 1983 e 1999, quando a difusão midiática sobre HIV era intensa; e a terceira, nos anos 2000 e 2010, com expressiva evolução científica sobre o manejo da condição e e o aumento da esperança de vida das pessoas vivendo com HIV.

Antes da graduação, portanto no inicio dos anos 1980, sua formação discursiva revela ausência ou escassez de informação sobre o tema devido a inexistência de relatos de casos em crianças.

Eu não tive experiência com HIV antes da graduação porque não existia HIV naquela época. (N6c, estudante de 1983-1987)

As enfermeiras que estudaram na década de 1980 vivenciaram o processo de descoberta dos primeiros casos e os esforços dos cientistas para compreender como o vírus operava no corpo humano. Documentos oficiais do Ministério da Saúde Brasileiro constavam nas referências dos programas de ensino das disciplinas das escolas de Enfermagem à época, informando professores e estudantes sobre descobertas, por vezes contraditórias e confusas. O ensino centrava-se mais nas normas de biossegurança do que sobre a condição das pessoas soropositivas para o HIV.

Na universidade, eu me lembro de um boletim do Ministério [da Saúde]... que o HIV era transmitido por mosquito. Alguns dias mais tarde, nós lemos que era impossível. Nós líamos e questionávamos tudo. “Transmite ou não?” Não havia informação. Ninguém sabia de nada. Quando estávamos em estágio, utilizavamos equipamento de segurança pessoal em excesso, especialmente para cuidar das crianças. Tudo era feito para proteger as crianças. (N6c) 
Nesse sentido, no processo de formação das enfermeiras, HIV/AIDS e biossegurança conformam um imaginário de medo de transmissão de uma doença letal que impregna a sua formação ideológica.

No final da década de 1980, houve os primeiros contatos de enfermeiras com familiares infectados pelo HIV e as primeiras crianças com AIDS. O único antiretroviral disponível no Brasil era o AZT, e o cuidado de enfermagem consistia em administrá-lo, implementar uma escuta autêntica e promover conforto e apoio.

Você se encontra com uma família extremamente humilde que não sabe o que está enfrentando. Naquela época (1989), AIDS dava ibope (era muito comentada publicamente), especialmente em crianças. Só existia o AZT. Então, não tinhamos muito o que fazer... conversavamos e seguravamos a mão. (N6C)

A pobreza material era resignificada como incapacidade cognitiva de compreender o que era HIV/AIDS. Estar infectado significava a morte e na infância repercutia no fenômeno da morte infantil pouco aceito socialmente. Ademais, o cuidado de conforto e a escuta foram resignificados como pouco importante na cura de uma doença que não tem cura. $O$ cuidado de enfermagem ouvir e promover conforto e apoio são resignificados como "nada muito a fazer".

Quatro enfermeiras (N1a, N7d, N10e, N11e), cuja graduação aconteceu após 1996 pertencem a um grupo de estudantes de enfermagem que tiveram contato com informações pela mídia de massa e aquelas disseminadas pelo Sistema Único de Saúde (SUS) no controle da epidemia. Na década de 1990, como parte da totalidade, as participantes estavam imersas em um contexto social em que pessoas famosas (Cazuza, Renato Russo, etc.) no Brasil, apresentavam-se fisicamente caquéticas e com manchas na pele, publicizaram sua condição de soropositividade para a sociedade. Isso contribuiu para construções sociais estereotipadas do físico de uma pessoa com AIDS.

As pessoas morriam. Cazuza, Renato Russo... E outras pessoas que eu não conhecia morreram. Aqueles que mostraram suas caras eram pessoas que pareciam cadáveres. Elas eram magras, andavam de cadeira de roda... E se elas eram socialmente ou economicamente privilegiadas, tinham cultura e educação; a morte chegava para elas também. (N5c, N7d, N8d, N11e, N12f)

A letalidade do HIV transcende as condições econômicas e de escolarização, porque pessoas economicamente mais privilegiadas morriam pela AIDS. A morte não discriminava, acontecia para todos, incluindo pessoas próximas das enfermeiras. Uma das participantes vivenciou o HIV dentro de sua família. 
O que eu associei com a minha experiência antes da faculdade foi a morte de meu primo diagnosticado com HIV. Era os anos 1990, as pessoas descobriam e morriam meses depois. (N13f)

Encontro com pessoas conhecidas refletiu em formações imaginárias impregnadas por estigma, preconceito e letalidade associada à essa condição de saúde. Reconheciam o HIV como uma ameaça séria que todos deveriam temer no seu cotidiano.

Antes da graduação, eu me lembro de uma família que vivia perto da minha casa. A mãe tinha HIV e tratava como se fosse gripe, não sabia da gravidade [do HIV]. (N2a)

Durante os anos 2000 a 2010, um grupo de seis enfermeiras (N2b, N3b, N4b, N5c, N8d, $\mathrm{N} 9 \mathrm{e}, \mathrm{N} 12)$ foi estudante em um momento que as ações governamentais, no enfrentamento da epidemia do HIV/AIDS, eram reconhecidas internacionalmente. Algumas enfermeiras associaram suas formações ideológicas de estigma, preconceito e letalidade, com a desinformação sobre o mecanismo patológico do HIV. Elas reconheceram essas construções em si e consideravam-se leigas antes de sua formação profissional.

Antes da faculdade, eu tinha preconceito. Eu não sabia como a patologia funcionava. Minha compreensão da doença era o mesmo de alguém leigo. Eu pensava que era doença da homossexualidade e da promiscuidade. (N4b, N5c, N7d, N8d, N10e)

\subsection{A formação profissional para o cuidado à criança com HIV/aids}

Mesmo durante o curso de graduação, o preconceito e o medo de contaminar-se permaneceram presentes na formação imaginária, se manifestando durante o processo de cuidar. Quando iniciaram o estágio clinico, resignificavam a pessoa com HIV como a doença em si. Mesmo tendo estudado na década de 2000, os procedimentos de biossegurança eram tão excessivos quanto àqueles relatados pela enfermeira que se graduou na década de 1980, com a mesma perspectiva de cuidado de enfermagem à pessoa com HIV/aids em um espaço de tempo de 20 anos.

Quando eu pensava em HIV, meu preconceito estava lá. Eu tinha medo de ficar perto daquelas pessoas, eu tinha medo do cuidado de enfermagem a pessoa com AIDS. Quando interagi com esse paciente, lembro de ter usado muito equipamento de segurança. Foi uma surpresa ver que $98 \%$ dos pacientes na unidade eram soropositivos para o HIV. Eu pensava: Deus, que mundo a gente vive? (N3b,N4b).

Discursos revelaram que no curso de graduação não houve a experiência do cuidado à criança com HIV/aids, porque o adoecer durante a infância é resignificado como tristeza e dor. Nesse sentido, para evitar o sofrimento da estudante, o professor a protege dessa realidade privando-a da experiência de cuidar. 
Eu não lembro de permitirem a gente interagir com a criança com HIV. Meus professores achavam ser muito complicado para a gente. Nós lidávamos com adultos, nunca interagimos com crianças, acho que os professores queriam nos proteger. Era triste vê-las doente. (N4b, N5c, N11f)

Por não ter investido na ruptura do preconceito durante o processo de formação, o preconceito continuou na prática profissional. Mesmo sem intencionalidade, há uma tensão no ambiente de cuidados clínicos.

Quando você sabe que a criança tem HIV, você se sente assim. Mesmo sem querer diferenciá-las, você diferencia. Você tem muito mais cuidado quando elas têm HIV. O ambiente fica mais tenso. (N1a, N2a)

A tensão reside no tipo de cuidado que é remodelado quando há uma hospitalização com HIV/aids, o ambiente de cuidado passa a ser diferenciado, mesmo sem que tenham percepção do preconceito. Na atenção primária, as enfermeiras resignificaram a gravidez como uma falha das ações dos profissionais de saúde que acompanhavam a adolescente com HIV na estratégia de saúde da família.

Havia uma adolescente na área de abrangência [da Estratégia de Saúde da Família] que eu trabalhava. E eu não sei o porquê ou onde nós falhamos, mas ela apareceu grávida. Foi um pré-natal bem difícil porque ela não tomava os antirretrovirais. Então eu pensava "por que uma pessoa assim engravida?" (N2a, N11e)

\subsection{Enfermeiras na revelação do HIV/aids à criança}

A revelação à criança é resignificada como um algo pontual, quando o médico pela primeira vez comunica o diagnóstico ao responsável.

Eu não vivenciei a revelação do diagnóstico. Eu devo confessar que acho muito complicado. Isso não é função do médico? (N1a, N2a, N3b, N4b, N5c, N7d, N8d, N9e, N10e, N11e, N12f, N13f).

Quanto a participação no processo de preparação da família para revelar a criança sua condição soropositiva, a maioria das enfermeiras desconhece sua competência legal nesse processo. Duas reconheceram o direito de a criança saber sobre sua condição de saúde.

Eu não participei diretamente, mas é importante que a criança conheça seu diagnóstico. É seu direito e é nosso trabalho garantir que ele seja respeitado nos espaços de saúde. (N5c, N8d)

Do ponto de vista pragnmático, há vantagem no processo terapêutico quando a criança sabe sobre sua soropositividade para o HIV. Os profissionais, familiares e criança têm mais liberdade para dialogar durante o atendimento no serviço de saúde. Há maior fluidez e 
abertura na comunicação, o que favorece a educação em saúde da criança para torná-la mais autônoma em seu cuidado.

Eu vejo a diferença quando elas sabem [o diagnóstico] porque eu posso conversar com elas sobre. Elas fazem perguntas, querem saber sobre o remédio, os exames... As meninas perguntam sobre a menstrução, como namorar, se podem ter filhos... Essas coisas (N5c)

Outra participante reconhece sua participação na revelação, mas não no preparo da família. Mesmo sabendo que a enfermeira tem competência legal para isso, refere que a carga de trabalho nem sempre permite esse envolvimento.

Eu participei. Eu lembro que uma mãe nos pediu para estar com ela quando ela contasse para o filho. Foi interessante e eu acho que as enfermeiras têm conhecimento para isso, mas requerem vínculo com a família e, nem sempre, conseguimos construí-lo durante o turno porque são muitas coisas para fazer. (N6c)

Um dos obstáculos resignificados pelas enfermeiras em participar ou não do processo é a falta de conhecimento sobre sua competência legal no contexto. Suas construções sobre preparo da família entrelaçam-se com a ideia de descoberta do diagnóstico médico.

Não me sinto confortável de falar sobre o assunto com as famílias. Acho difícil saber o que posso ou não falar, não é trabalho do médico? Eu preciso cuidar de outras demandas como ensiná-los a engolir o remédio ou orientar os pais sobre consultas e adesão ao tratamento. (N6c, N8d, N9e, N11e, N12f)

Apesar de as enfermeiras reconhecerem o direito da criança de acesso à infomação sobre sua saúde, as formações ideológicas e imaginárias do estigma e preconceito são estruturais. O sentido do HIV/aids na infância como uma doença letal não é condizente com os tempos da doença AIDS como uma condição crônica. A ideologia biomédica transversaliza o processo de revelação e o preparo de familiares para essa revelação à criança não é resignificado como um cuidado de enfermagem.

\section{DISCUSSÃO}

O processo discursivo desvelou formações ideológicas e imaginárias de enfermeiras que trabalham com crianças com HIV e suas famílias que refletem construções histórico-sociais do HIV, AIDS e infância. O movimento metafórico acerca da AIDS teve início na década de 1980, quando os primeiros casos foram divulgados na imprensa mundial e brasileira. No ano de 1983, uma das participantes da pesquisa iniciou o curso de graduação em Enfermagem, mesma época que um dos principais jornais brasileiros publicou um artigo intitulado "a peste gay", em que a AIDS é descrita como a pior doença de todos os tempos (Soares, 1998; 
Darde, 2004). O termo "peste" está relacionado às características de fatalidade e transformação corporal, mas na AIDS, ainda associava-se à vergonha que culpabilizava o indivíduo pela sua condição de saúde (Sontag, 1989).

As enfermeiras tiveram contato com esse tipo de linguagem midiática, no início da epidemia da aids no país, o que contribuiu para a construção do estereótipo que permaneceu como parte constitutiva de suas formações imaginárias. Mesmo que o estigma e preconceito com o HIV se manifestem de forma sutil e inconsciente, com expressões de medo, "não há mais o que fazer", indicando há interferência na qualidade do cuidado prestado. Entre as manifestações destacam-se olhares com nuances significativas, atitudes movidas pelo medo, perguntas incovenientes, conversas desconfortáveis e até recusa de assumir um determinado usuário (Salih et al., 2017; Reys-Estrada, Varas-Díaz, \& Martínez-Sarson, 2015).

A linguagem é uma importante aliada no enfrentamento do preconceito e estigma estrutural no HIV/AIDS (Chambers et al., 2015). É por meio das palavras que os significados sociais se desconstroem e a enfermeira, enquanto parte da equipe de saúde pode atuar no cotidiano de preparo da família para revelação do HIV à criança soropositiva. Contudo, a centralização do cuidado em procedimentos é um dos desafios à implementação de uma abordagem que valorize a escuta autêntica e orientação antecipada. A escuta autêntica favorece a comunicação com a família para conhecer sua história e compreender como ela vivencia a condição de saúde. Já o cuidado de advocacia envolve o papel da enfermeira enquanto defensora do direito da criança para participar do seu cuidado de saúde que começa com o acesso à informação sobre sua condição sorológica (Bubadué \& Cabral, 2019; Koerich et al., 2015; Våga, Moland, \& Blystad, 2016).

As construções idelógicas das enfermeiras acerca do HIV, da infância e de como compreendem cuidado de enfermagem interferem na maneira que abordam o tema com a família que se prepara para revelar o HIV da criança a ela. Utiliza-se HIV, uma vez que utilizar nomenclaturas como doença ou condição reforça o silêncio fundado acerca do tema, contribuindo para o estigma e preconceito de nomear o HIV. Apesar de reconhecerem o direito da criança de acesso à informação de sua saúde, formações imaginárias centradas no paradigma biomédico ainda deixam as enfermeiras inseguras quanto à sua competência legal diante desse fenômeno. Em contrapartida, pesquisas demonstram que a família indica a enfermeira como uma profissional competente que participa no processo de seu preparo 
para conversar com a criança sobre o HIV dela (Bubadué \& Cabral, 2019; Simoni et al., 2015; Finnegan et al., 2019).

As intervenções de enfermagem apontadas como aliadas no processo de preparo da família foram a escuta autência, a orientação antecipada, o cuidado de advocacia e o acompanhamento no pós-revelação (Simoni et al., 2015; Våga, Moland, \& Blystad, 2016; Bubadué \& Cabral, 2019; Finnegan et al., 2019).

\section{CONCLUSÕES}

As enfermeiras construíram interseções da aprendizagem informal na vida cotidiana com os estudos formais e a vida profissional de cuidar de crianças com HIV/aids e o drama da revelação da condição soropositiva a criança. Suas formações ideológicas revelaram o HIV/AIDS como um tema sensível porque é marcado pelo estigma e preconceito estrutural. Em contrapartida, reconhecem o direito da criança saber sobre sua condição de saúde. Nesse contexto o preparo da família emerge como um direito da criança de acesso à informação segura e com linguagem que favoreça o processamento dessa informação de maneira mais clara e atraumática possível.

Os desdobramentos da pesquisa ocorreram no processo reflexivo da prática profissional das participantes do estudo, bem como a continuidade da pesquisa com a criação de curtametragem sobre o preparo das famílias para revelar o HIV à criança pelas enfermeiras.

Como limitação do estudo tem-se sua aplicabilidade ao contexto do setor público do Rio de Janeiro, necessitando desenvolver pesquisas com esse desenho em outros cenários.

Financiamento. Os autores foram financiados pela Coordenação de Aperfeiçoamento de Pessoal de Nível Superior (CAPES) nas modalidades de Bolsa de Doutorado (2015-2019) e Bolsa Produtividade 2 , respectivamente.

\section{REFERÊNCIAS}

Adedimeji, A., Edmonds, A., Hoover, D., Shi, Q., ... Yotebieng, M. (2017). Characteristics of HIV-Infected Children at Enrollment into Care and at Antiretroviral Therapy Initiation in Central Africa. PloS one, 12(1), e0169871. doi:10.1371/journal.pone.0169871

Archibald, M. M., Caine ,V., \& Scott, S.D. (2017). Intersections of the arts and nursing knowledge. Nurs Inq., 24(2). doi: 10.1111/nin.12153. Epub 2016 Aug 30.

Brasil. Ministério da Saúde. Secretaria de Vigilância em Saúde. Departamento de Vigilância, Prevenção e Controle das Infecções sexualmente trasnmissíveis, do HIV/AIDS, das Hepatites Virais. (2017). Clinical 
Protocol and Therapeutic Guidelines for Handling HIV infection in children and adolescents. Brasília: Ministry of Health.

Brasil. Ministério da Saúde. Secretaria de Vigilância em Saúde. Departamento de Vigilância, Prevenção e Controle das Infecções Sexualmente Transmissíveis, do HIV/Aids e das Hepatites Virais. (2018). Technical Manual for the Diagnosis of the HIV Infection in Adults and Children. Brasília: Ministry of Health.

Bubadué, R. M., \& Cabral, I. E. (2019). Advocacy Care on HIV disclosure to Children. Nursing Inquiry, 26(2), e12278.

Cabral, I. E, \& Neves, E. T. (2016). Pesquisar com o método criativo e sensível na enfermagem: fundamentos teóricos e aplicabilidade. In Lacerda, M. R, \& Costenaro, R. G. S. (Orgs). 1 ed. Porto Alegre: Editora Moriá. pp. 325-50.

Caldas, A. C. S., Dias, R. S., Sousa, S. M. A., \& Teixeira, E. (2019). Creative and sensitive production of careeducational technology for families of children with gastrostomy. Escola Anna Nery, 23(1), e20180144.https://dx.doi.org/10.1590/2177-9465-ean-2018-0144

Chambers, L. A., Rueda, S., Baker, D. N., Wilson, M. G., ... Stigma Review Team (2015). Stigma, HIV and health: a qualitative synthesis. BMC public health, 15, 848. https://doi.org/10.1186/s12889-015-2197-0

Darde, V. W. S. (2004). A AIDS na imprensa: a construção da imagem da epidemia e a influência na promoção da cidadania. Em Questão, 10(2), p. 247-259.

Finnegan, A., Langhaug, L., Schenk, K., Puffer, E.S., ... Green, E.P. (2019). The prevalence and process of pediatric HIV disclosure: A population-based prospective cohort study in Zimbabwe. Plos One. https://doi.org/10.1371/journal.pone.0215659

Freire, P. (2006). Pedagogy of the oppressed. 30 ed. New York : The Continuum International Publishing Group Inc.

Gyamfi, E., Okyere, P., Appiah-Brempong, E., Adjei, R. O., \& Mensah, K. A. (2015). Benefits of Disclosure of HIV Status to Infected Children and Adolescents: Perceptions of Caregivers and Health Care Providers. J Assoc Nurses AIDS Care, 26(6), 770-80. doi: 10.1016/j.jana.2015.08.001. Epub 2015 Aug 10.

McNiff, S. (2007). Art Based Research. In Knowles, J. G. Handbook of the Arts in Qualitative Research: Perspectives, Methodologies, Examples, and Issues. United States of America: Sage publications.

Orlandi, E. P. (2013). Análise de discurso: princípios e procedimentos. 11 ed. Cmapinas: Pontes.

Partelli, A., Cabral, I. E. Images of alcohol in the adolescents' life of one quilombola community. Revista Brasileira de Enfermagem, 72(2), 468-475. Epub April 18, 2019.https://dx.doi.org/10.1590/0034-7167-2018-0264

Potrich, T., Paula, C. C., Padoin, S. M. de M., \& Gomes, A. M. T. (2016). Cotidiano do familiar no cuidado à criança com HIV em tratamento antiretroviral. Rev enferm UERJ, 24(4), e17446.

Reyes-Estrada, M., Varas-Díaz, N., \& Martínez-Sarson, M. T. (2015). Religion and HIV/AIDS Stigma: Considerations for the Nursing Profession. The New School psychology bulletin, 12(1), 48-55.

Salih, M. H., Tessema, G. A., Cherkos, E. A., Ferede, A. J., \& Anlay, D. Z. (2017) Stigma towards People Living on HIVIAIDS and Associated Factors among Nurses' Working in Amhara Region Referral Hospitals, Northwest Ethiopia: A Cross-Sectional Study. Advances in Nursing. https://doi.org/10.1155/2017/6792735

Sariah, A., Rugemalila, J., Somba, M., Minja, A., ... Siril, H. (2016). Experiences with disclosure of HIV-positive status to the infected child: Perspectives of healthcare providers in Dar es Salaam, Tanzania. BMC public health, 16(1), 1083. doi:10.1186/s12889-016-3749-7

Simoni, J. M., Yang, J. P., Shiu, C. S., Chen, W. T., ... Lu, H. (2015). Nurse-delivered counselling intervention for parental HIV disclosure: results from a pilot randomized controlled trial in China. AIDS (London, England), 
29(Suppl 1), S99-S107. https://doi.org/10.1097/QAD.00000000000000664

Soares, R. L. (1998). Aids e imprensa: escritos do jornal Folha de S. Paulo. Interface - Comunicação, Saúde, Educação, 2(2), 47-76. https://dx.doi.org/10.1590/\$1414-32831998000100004

Sontag, S. (1989). Aids e suas metáforas; trad PH Brito. São Paulo: Companhia das Letras.

Soratto, J., Pires, D. E. P., Cabral, I. E., Lazzari, D. D., ... Sipriano, C. A. S. (2014). A creative and sensitive way to research. Revista Brasileira de Enfermagem, 67(6), 994-999. https://dx.doi.org/10.1590/00347167.2014670619

UNICEF. United Nations Children's Fund. (2019). Annual Report: 2018. For every child, every right. New York.

Våga, B. B., Moland, K. M., \& Blystad, A. (2016). Boundaries of confidentiality in nursing care for mother and child in HIV programmes. Nursing Ethics, 23(5), 576-586. https://doi.org/10.1177/0969733015576358

Van Elsland, S. L., Peters, R., Grobbelaar, C., Ketelo, P... van Furth, A. M. (2019). Disclosure of human immunodeficiency virus status to children in South Africa: A comprehensive analysis. Southern African journal of HIV medicine, 20(1), 884. doi:10.4102/sajhivmed.v20i1.884

WHO. World Health Organization. Guideline on HIV disclosure counselling for children up to 12 years of age. (2011). Switzerland: Beatrice Krauss, Susan Letteney, Anniek De Baets, Julie Murugi andF. Amolo Okero.

Wright, S., Amzel, A., Ikoro, N., Srivastava, M., ... Phelps B. R. (2017) Talking to children about their HIV status: a review of available resources, tools, and models for improving and promoting pediatric disclosure. AIDS Care, 29(8), p. 1019-25.10.1080/09540121.2016.1273471 